\title{
Dynamical triggering of starbursts
}

\author{
F. Combes \\ LERMA, Observatoire de Paris, 61 Av. de l'Observatoire, F-75014, Paris, France
}

\begin{abstract}
Galaxy interactions/mergers, gravitational instabilities and density waves, such as bars, are frequently invoked to trigger starbursts. These mechanisms have been explored through numerical simulations, with the help of various star formation recipes. Gravitational instabilities are necessary to initiate star formation, but the main trigger might be the gas flows, to provide sufficient fuel in a short time-scale. Gas accretion is also acting on the dynamics, in favoring bars/spirals, which will drive the gas inwards. Large amounts of external gas accretion are required to explain the bar frequency, and this accretion rate can be provided by the cosmic filaments, as supported by cosmological simulations. Subsequent interactions can then trigger starbursts by driving this accreted gas inwards.
\end{abstract}

\section{OBSERVATIONAL EVIDENCE OF DYNAMICAL TRIGGERING}

It is now widely accepted that strong galaxy interactions can produce violent starbursts, and ultra-luminous infra-red galaxies (ULIRGs) are all mergers (e.g. Sanders \& Mirabel 1996). But interacting galaxies in general do not show intense starbursts (Bergvall et al 2003), or only in their centers. The tidal parameter, depending on mass and distance of companions, is not correlated to any star formation tracer. Starbursts are rare in the local universe, and much rarer than galaxy interactions. The only perceptible effect is that interacting galaxies have more mass - gas, dust \& young stars, and are more concentrated to the center (Bergvall et al 2003). One of the main conclusions that can be drawn for giant galaxy starbursts is that galaxy interactions is a necessary condition to trigger them, but not sufficient. Certainly, other parameters have to be taken into account, such as the gas content, the geometry/velocity of the encounter, and also the phases of the interaction, the starburst occuring only during about $10 \%$ of the interaction time, according to simulations (e.g. Mihos \& Hernquist 1996).

For dwarf galaxies, interactions are even not a necessary condition for triggering: starbursting dwarfs have no more companions (Brosch et al 2004), although it might be different for Blue Compact Dwarfs (BCD, Hunter \& Elmegreen 2004). Dwarf galaxies with starbursts present asymmetries, and a possible trigger could just come from gas accretion: the gas infalling in dark-matter haloes may experience sloshing and oscillations favoring compression and instabilities. This mechanism might be relevant for primordial galaxies (Brosch et al 2004).

One of the best evidence of triggering can be obtained by tracing the fossil records of the star formation history in nearby galaxies. In the Small Magellanic Cloud, the number of stars at a given age has been determined by Zaritsky \& Harris (2004). They noticed that the derived SMC star formation history reveals some bursts corresponding to pericenters of its orbit around the Milky Way. A model taking the tidal triggering 


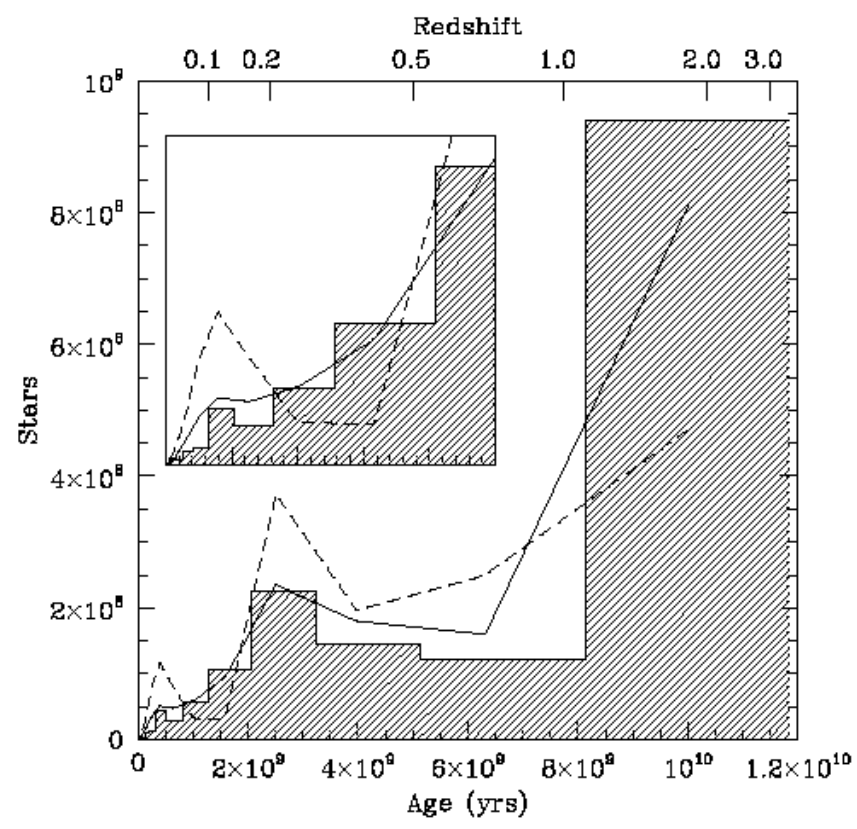

FIGURE 1. Star formation history in the SMC derived from a tidally triggered model (dash-line), or a tidally triggered model with infall (solid line), compared to the observed number of stars at each age, from Zaritsky \& Harris (2004). The insert is a zoom of the recent 2.5 Gyr.

into account fits better the observations, although the quantitative influence of the tide is uncertain, between 10 and $70 \%$ of the star formation could be tidal in origin, cf figure 1] One of the problem in the fit comes from the too large increase in star formation about 3-4 Gyrs ago, which can only be explained by external gas accretion. A significant gas infall, by about $50 \%$ of the total gas mass, improves the fit, and accounts also for the observed age-metallicity relation. The metallicity is not monotonously increasing with decreasing age, but remains constant for old stars until 3 Gyrs, indicating that dilution is required from deficient gas infall.

\section{DYNAMICAL PROCESSES}

The star formation rate in spiral galaxies is observed to be proportional to the power $\mathrm{n}=1.5$ of the gas density, corresponding empirically to a global Schmidt law over the galaxy (Kennicutt 1998), although the local Schmidt law is not observed. The SFR law is the same for interacting and non-interacting galaxies, whatever their triggers are. This law has been interpreted through several processes: Jeans instability can lead to this exponent, star formation being proportional to the gas density $\rho$ over the free-fall time scale, scaling in $\rho^{1 / 2}$, resulting in the power $3 / 2$, but also cloud-cloud collisions (Elmegreen 1998), or star-forming contagion with feedback, leading to chaotic conditions.

When strong starbursts are observed, they are in general concentrated in the central 
regions, and large amounts of gas must be fueled towards the center in a short enough time to beat the feedback. The radial gas flows can be due to bars, or spiral density waves. Molecular gas concentrations are observed in barred galaxies (e.g. Sakamoto et al 1999), and circumnuclear starbursts are frequently found in resonant rings (e.g. Buta \& Combes 1996).

\section{Star formation efficiency}

The star formation efficiency (or SFE) can be defined by the fraction of gas consumed in star formation per dynamical time. In normal galaxies, the SFE is about 0.1-3\%, but in some interacting galaxies and ULIRGs, it can be as high as 50\%. The star formation rates in spiral galaxies range from 0.1 up to $1000 \mathrm{M}_{\odot} / \mathrm{yr}$, depending on mass, but SF can be about 100 times more efficient, in triggered galaxies.

The far infrared emission from dust L(FIR) is the best tracer of SF in enshrouded starbursts, and the gas content is traced by the $\mathrm{CO}$ emission. The dense gas tracers $(\mathrm{CS}$, $\mathrm{HCN}$ ) appear to vary linearly with L(FIR), and can be used also as SF tracer (Solomon et al 1992, Gao \& Solomon 2004). While the SFE (FIR to CO ratio) may vary by a factor 100 , the $\mathrm{HCN} / \mathrm{CO}$ emission ratio varies also in the same proportion, meaning that in average the gas is denser in starbursts. The HCN/FIR ratio corresponds to star forming cores in the Milky Way, and the ULIRGs can be modelled with a large filling factor of SF cores. May be the necessary step to trigger a starburst is to form the dense cores, and then the efficiency per unit core mass is universal (Gao \& Solomon 2004, Fritze v. Alvensleben 2004).

\section{Other dynamical processes}

When two galaxies interact and their disks overlap, there could be violent collisions between clouds at velocities of the order of $300 \mathrm{~km} / \mathrm{s}$; diffuse HI clouds collide, but molecular GMCs not, due to their small filling factor. However, they are compressed by the created hot high pressure medium (Jog \& Solomon 1992). Also, while GMCs are radially moving towards the center, they are compressed, provided that the ICM pressure increases with decreasing radius (Jog \& Das 1996). From stability arguments, if the Toomre Q-parameter maintains constant over the whole disk, the velocity dispersion and therefore the gas turbulence is higher in the center: the critical velocity dispersion $\mathrm{V}_{\text {crit }}(=3.36 \mathrm{G} \Sigma / \kappa)$ decreases with radius for constant rotation curves.

Similarly, in clusters, star formation could be induced by the compression of GMCs by the intra-cluster hot gas (Bekki \& Couch 2003). This might explain the starbursts occuring in the spiral galaxies infalling into the cluster Abell 1367 (Gavazzi et al 2003).

\section{STAR FORMATION RECIPES}

Numerical simulations of galaxies use recipes for star formation, as all sub-grid physics (Katz 1992; Mihos \& Hernquist 1994). These can be based on the Schmidt law, or the Jeans instability, with some criteria taken into account, like density thresholds, gas temperature, convergent gas flows. These recipes can simulate the dynamical triggering; simulated galaxy interactions produce strong non-axisymmetry and torques that drive 

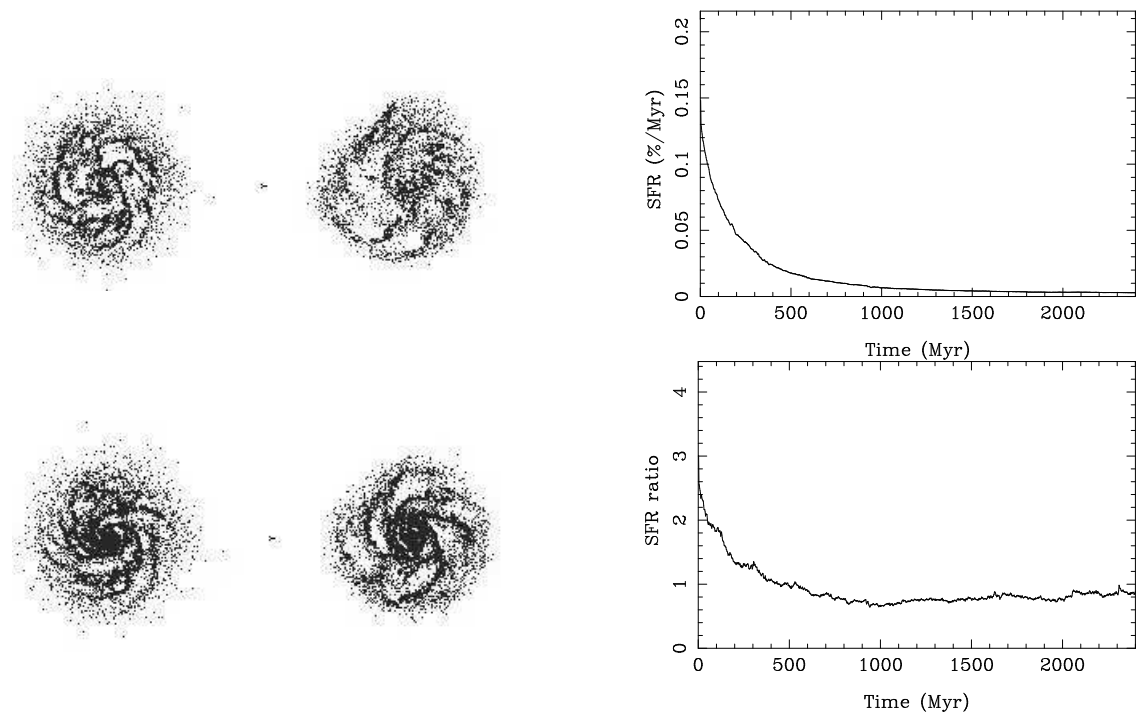

FIGURE 2. Comparison of two simulations of isolated galaxies, with different Q-parameter for the gas (different initial velocity dispersions). Top Left: gas morphology during the two first epochs of the simulation with $\mathrm{Q}_{g}=0.3$, separated by $200 \mathrm{Myr}$; Right: corresponding SFR (fraction of the gas transformed into stars per Myr) as a function of time. Bottom Left: same for the simulation with $\mathrm{Q}_{g}=0.8$; Rigth: Ratio of the SFR in these two simulations, as a function of time. At the beginning, the star formation rate is 3 times higher, for $\mathrm{Q}_{g}=0.3$.

the gas towards the center. The strength of these perturbations depends essentially on the stability of the disk prior the interaction, therefore on the bulge-to-disk ratio, while the role of the orbital geometry appears secondary, provided the interaction ends in a merger (Mihos \& Hernquist 1996). However, many parameters involving the gas physics or the stellar feedback can influence significantly the SFR, while they are still uncertain.

\section{Influence of gas stability}

The small scale structure of the gas, depending on its surface density in the galaxy, and its velocity dispersion, has a large influence on the SFR, modifying the fraction of dense clumps. Figure 2 illustrates how a small variation of initial $\mathrm{Q}_{g}$ of the gas can multiply the SFR by a factor 3 .

The influence of the gas velocity dispersion can also be included, to represent the shock-induced star formation in interacting galaxies, since cloud-cloud collisions might be involved in the mechanism. A modified Schmidt law in $\rho^{n} \sigma^{\beta}$ to take into account the velocity dispersion $\sigma$ has been introduced by Brosche \& Lentes (1985). It was applied by Barnes (2004) to the Mice simulations, and revealed a better match of the observations, with the exponent $\beta=0.5$.

\section{Influence of multi-phase gas, feedback}

Hydro-SPH simulations ignore the various phases, and smooth the density contrast: hot diffuse gas is prevented to exist together with dense cold clumps; the dense gas evaporates, while the hot gas cools exaggerately. Marri \& White (2003) attempt to separate two gas phases in the SPH code (with neighbor particles searched separately) 
taking into account mass exchange between the two phases: this of course changes significantly the star formation history of a spiral galaxy. The star formation should also be limited by stellar feedback, which is phenomenologically taken into account through mass loss and energy reinjected locally around star-forming regions. However the largescale fountain effect, or galactic winds, and large-scale outflows are not simulated correctly (Thacker \& Couchman 2001).

\section{IMPORTANCE OF GAS INFALL}

Many observations point towards an almost stationnary star formation history in spiral galaxies for intermediate Hubble types (Kennicutt et al 1994, Brinchmann et al 2004). However, even taking into account the stellar mass loss, an isolated galaxy should have an exponentially decreasing star formation rate. To fuel the star formation, galaxies depend on external accretion. Mergers with gas rich dwarf companions are not sufficient; for instance all objects falling now on the Milky Way represent less than 1/400 of the mass of the Galaxy (Ibata et al 2001). Galaxies must accrete large amounts of gas mass along their lives (Brinchman et al 2004).

The analysis of stellar populations in a large sample of galaxies (SDSS, Heavens et al 2004) have shown that massive galaxies have formed most of their stars at early times, while dwarf galaxies are still forming now. Only galaxies of intermediate masses could have in average maintained their star formation rate over a Hubble time.

\section{Simulations in a cosmological context}

When simulated inside the filamentary cosmic web of gas and dark matter, the star formation rate of galaxies is no longer exponential. The star formation is both fueled by galaxy mergers, and in the mean time, external gas accretion (e.g. Tissera 2000, Nagamine et al 2004). One of the essential role of companions is to trigger star formation, by driving this accreted gas inwards. Semi-analytical simulations, constrained by the luminosity function of galaxies and the Tully-Fisher relation, also derive an almost constant SFH for middle types (Somerville \& Primack 1999).

\section{Constraints from bars and spirals}

Recently, the bar frequency in nearby galaxies have been quantified from near-infrared surveys (e.g. Block et al. 2002), and is much higher than expected, given the destruction of bars in spiral galaxies with interstellar gas (Bournaud \& Combes 2002). To explain the observations, bars have to be reformed, and this is possible only through continuous gas accretion at a large rate. The observed bar frequency, combined with the prediction of dynamical models, allows to quantify the accretion rate (Block et al. 2002). An intermediate type spiral galaxy must double its mass in about $10 \mathrm{Gyr}$. Cold gas accretion is required to increase the self-gravity of the disk, and decrease the bulge-to-disk ratio. Dwarf companions accretion cannot play this role, since they heat the disks. Also major mergers develop the spheroids. Instead the filaments in the near environment of galaxies are the source of continuous cold gas accretion. The accretion rate derived in 
cosmological simulations is compatible with the mass doubling in 10 Gyr. Cosmological accretion can explain bar reformation.

\section{CONCLUSIONS}

Star formation depends essentially on the gas supply and is only slightly influenced by the large-scale dynamics. Galaxy interactions act to drive the accreted gas radially inwards and trigger central starbursts. The accretion of gas not only regulates the star forming history in galaxies but also their dynamical state (bars, spirals, warps, $m=1 \ldots$ ), and both phenomema interact in a feedback loop. For galaxies in the field, accretion is dominant, and explain bars and spirals, and the constant star formation rate for intermediate types. In rich environments, galaxies experience quicker evolution, mergers are much more important, and secular evolution of galaxies through gas accretion is halted at $\mathrm{z} \sim 1$, since galaxies are stripped from their gas reservoirs.

\section{REFERENCES}

Barnes J.E.: 2004, MNRAS 350, 798

. Bekki K., Couch W.: 2003 ApJ 596, L13

. Bergvall N., Laurikainen E., Aalto S.: 2003, A\&A 405, 31

. Block D., Bournaud F., Combes F., Puerari I., Buta R.: 2002, A\&A 394, L35

- Bournaud F., Combes F.: 2002, A\&A 392, 83

- Brinchmann, J., Charlot, S., White, S. D. M. et al.: 2004, MNRAS 351, 1151

. Brosch, N., Almoznino, E., Heller, A. B.: 2004, MNRAS 349, 357

. Brosche P., Lentes F.T.: 1985 A\&A 153, 157

. Buta R., Combes F.: 1996, Fund. Cosmic Phys. 17, 95

. Elmegreen B.G.: 1998, in "Origins of Galaxies" ed. C.E. Woodward et al. (astro-ph/9712352)

. Fritze-V. Alvensleben, U., 2004, in "Young Local Universe", Rencontres de Moriond, Eds: A. Chalabaev, T. Fukui, T. Montmerle, and J. Tran-Thanh-Van, astro-ph/0407360

. Gao Y., Solomon P.M.: 2004 ApJ 606, 271

- Gavazzi, G., Cortese, L., Boselli, A. et al.: 2003, ApJ 597, 210

. Heavens, A., Panter, B., Jimenez, R., Dunlop, J.: 2004, Nature 428, 625

. Hunter D.A., Elmegreen B.G.: 2004, AJ 128, in press (astro-ph/0408229)

. Ibata, R., Irwin, M., Lewis, G.F., Stolte, A.: 2001 ApJ 547, L133

. Jog, C. J., Das M.: 1996 ApJ 473, 797

. Jog, C. J., Solomon, P. M.: 1992, ApJ 387, 152

- Katz, N.: 1992 ApJ 391, 502

. Kennicutt R.C., Tamblyn, P., Congdon, C. E.: 1994 ApJ 435, 22

. Kennicutt R.C.: 1998, ARAA 36, 189

. Marri, S., White S.D.M.: 2003 MNRAS 345, 561

. Mihos J.C., Hernquist L.: 1994 ApJ, 437, 611

. Mihos, J.C., Hernquist, L.: 1996, ApJ, 464, 641

. Nagamine K., Springel, V., Hernquist, L.: 2004, MNRAS 348, 435

. Sakamoto K., Okumura S.K., Ishizuki S., Scoville N.Z.: 1999 ApJ 525, 691

- Sanders D., Mirabel I.F.: 1996, ARAA 34, 749

- Solomon, P. M., Downes, D., Radford, S. J. E.: 1992, ApJ 387, L55

- Somerville, R. S., Primack, J. R.: 1999, MNRAS 310, 1087

- Thacker R.J., Couchman H.M.P.: 2001 ApJ 555, L17

. Tissera P.: 2000, ApJ 534, 636

. Zaritsky D., Harris J.: 2004, ApJ 604, 167 